\title{
Introduction to the Special Issue on Streaming Media
}

A DVANCES in computers, networking, and communications have created new distribution channels and business opportunities for the dissemination of multimedia content. Streaming audio and video over networks such as the Internet, local area wireless networks, home networks, and commercial cellular phone systems has become a reality and it is likely that streaming media will become a mainstream means of communication. Despite some initial commercial success, streaming media still faces challenging technical issues, including quality of service (QoS) and cost effectiveness. For example, deployments of multimedia services over $2.5 \mathrm{G}$ and $3 \mathrm{G}$ wireless networks have presented significant problems for real time servers and clients in terms of high variability of network throughput and packet loss due to network buffer overflows and noisy channels. New streaming architectures such as peer-to-peer (P2P) networks and wireless ad hoc networks have also raised many interesting research challenges.

This Special Issue is intended to address some of the principal technical challenges for streaming media by presenting a collection of the most recent advances in research and development that take into account aspects of both the media source and network communication, and/or provides an analysis and optimization of streaming systems.

Following an open call for papers, we received a total of 73 submissions for this Special Issue, spanning all topics in streaming media. Due to page limitations, we have had to turn away many fine quality papers. To ease the situation, papers by the guest editors were not included in this Special Issue. After an extensive and very competitive review process, we selected 16 papers for final publication. Some papers that were not selected for publication may appear in future regular issues of the IEEE TRAnsactions ON Multimedia. The papers in this Special Issue are organized into four sections: Error Resilient Streaming, Adaptive and Wireless Streaming, Novel Streaming Architectures and Protocols, and Efficient Streaming Media Distribution.

The first four papers deal with Error Resilient Streaming. The papers "Globally optimal uneven error-protected packetization of scalable code streams," by Dumitrescu, Wu, and Wang, and "Efficient channel code rate selection algorithms for forward error correction of packetized multimedia bitstreams in varying channels," by Stankovic, Hamzaoui, and Xiong, both provide algorithms for determining how much redundancy to add to packetized media, when using erasure coding across the packets to deal with packet loss, and/or error correction coding within each packet to deal with packet corruption. The objective of the algorithms is to select the rates of the constituent codes to minimize the expected distortion or maximize the expected fidelity

Digital Object Identifier 10.1109/TMM.2004.824219 of the recovered media, given the number of packets $N$ and the packet size $L$, as well as the source and channel statistics. Dumitrescu et al. provide a relatively efficient $O\left(N^{2} L^{2}\right)$ dynamic programming algorithm for finding an optimal unequal error protection (UEP) solution in the general case, while Stankovic et al. provide an ultrafast $O(N)$ algorithm for finding an optimal equal error protection (EEP) solution in the case where each packet contributes independently to the fidelity. The papers "Wyner-Ziv coding of video: An error-resilient compression framework," by Sehgal, Jagmohan, and Ahuja, and "Isolated regions in video coding," by Hannuksela, Wang, and Gabbouj, both provide methods for limiting error propagation due to lost packets in predictive video coding. Sehgal et al. use a small amount of parity information to ensure that every $m$ th intercoded frame can be recovered without error, thus limiting error propagation to at most $m$ frames. The authors show that this is much more efficient than using intracoded frames for this purpose. Hannuksela et al.introduce isolated regions, which are spatio-temporal structures akin to highly flexible sequences of slices. By disallowing prediction of one isolated region from another, error propagation is limited to each region. The authors show how to use these regions in the presence of packet loss for mode selection, unequal error protection, random access, and scene mixing.

The second set of four papers deals with Adaptive and Wireless Streaming. The paper "Streaming video over variable bit-rate wireless channels," by Stockhammer, Jenkač, and Kuhn, addresses the issue of successful on-time video decoding and presentation in the scenario of streaming over variable bit rate (VBR) wireless channels. The minimum startup delay and the minimum required receiver buffer size for a given video bitstream and a deterministic VBR channel are derived. Theoretical bounds for the startup delay and receiver buffer size are also derived that allow achieving certain quality of service for random VBR wireless channels. The paper "Providing adaptive QoS to layered video over wireless local area networks through real-time retry limit adaptation," by $\mathrm{Li}$ and van der Schaar, proposes a cross-layer protection method that can provide adaptive quality of service to layered coded video for improved performance of video streaming over wireless local area networks. This is achieved by incorporating prioritized delivery service using a priority queuing mechanism enhanced with some packet filtering and purging functionalities, and by unequal protection using adaptive MAC layer retransmission limits to balance the link erasure rate and buffer overflow rate. The paper "Seamless switching of scalable video bitstreams for efficient streaming," by Sun, Wu, Li, Gao, and Zhang, proposes a novel seamless switching scheme that switches among multiple scalable video bitstreams to efficiently accommodate channel bandwidth variations during streaming. While small bandwidth variations are accommodated by the scalability of 
single streams, large fluctuations generate switching between different bitstreams. A novel coding scheme is proposed to enable drift-free switching to a smaller rate stream, while a "switching frame" coding scheme allows jumping onto higher rate stream. The last paper, "Double feedback streaming agent for real-time delivery of media over 3G wireless networks," by Cheung, Tan, and Yoshimura, considers a system where a server in the wired network is delivering multimedia data to a client in a wireless network. Noting that the wired and wireless parts of the network have very different characteristics, the authors propose the use of an agent at the junction of wired network and wireless link to provide additional feedback information so that the system is not constrained to operate at the minimum of the two rates allowed by wired and wireless parts, and to enable various forms of error control mechanisms at the agent. The authors demonstrate the potential benefits of their proposed technique in several scenarios with different end-to-end delays.

The third set of four papers explores Novel Streaming Architectures and Protocols. The paper "Multiple sender distributed video streaming," by Nguyen and Zakhor, studies a receiver-driven protocol for simultaneous video streaming from multiple senders to a single receiver. In particular, this work focuses on two key components of such a system. First, the authors consider a rate allocation scheme that determines based on current network conditions the sending rate for each of the senders. Second, a packet partition algorithm is designed that ensures that any given video packet is sent by only one of the senders. The paper "Quality monitoring of video over a packet network," by Reibman, Vaishampayan, and Sermadevi, considers methods to monitor the quality of video being transmitted over a network without having access to the original video data, allowing a network service provider to evaluate the impact of packet loss on video stream quality. The authors propose three techniques that have increasing levels of bitstream parsing. Simulation results with MPEG-2 show that these techniques can approximate the true distortion, with particularly accurate estimates being available when the maximum level of bitstream parsing is enabled. The paper "Placement of continuous media in wireless peer-to-peer networks," by Ghandeharizadeh, Krishnamachari, and Song, explores a novel streaming architecture that consists of collaborating home-to-home online devices to provide on-demand video delivery. The authors propose a novel media data replication technique that enhances the startup latency while minimizing the total storage space, and discuss its implementation in both a centralized and a decentralized manner. In the paper "Peer-to-peer based multimedia distribution service," Xiang et al. propose a novel framework for multimedia distribution service based on P2P network. To achieve QoS awareness, the framework builds a topology-aware overlay. In such a network, the peers self-organize themselves, and are clustered into application groups. The requested content is distributed in two ways: if the requested content is within the same group, the content is delivered directly to the requestor; otherwise, a controlled flooding search algorithm finds the content at the group level. To improve system performance such as service request availability or latency, the authors investigate effectiveness of inter-group-replication and intra-group-replication strategies. The results show that group-replication schemes perform much better in terms of average delivery latency, PSNR video quality, and availability of requested content, as compared to the FreeNet system and random replication system.

The last four papers address the problem of Efficient Streaming Media Distribution. They respectively tackle placement of replica in scalable content distribution systems, proxy cache allocation, proxy-based streaming to heterogeneous clients, and broadcast of media data with time-varying demand. The paper "Minimizing delivery cost in scalable streaming content distribution systems," by Almeida, Eager, Vernon, and Wright, proposes to minimize the total server and network delivery cost in a scalable content distribution system, by efficient placement of a carefully chosen number of replica servers, and optimal routing of client requests and multicast streams. The authors solve this complex optimization problem for various client populations and realistic network topologies, and derive helpful insights and efficient heuristics to design large and heterogeneous systems, that are of practical interest. The best approximate heuristic produces systems with total delivery cost that is within $16 \%$ of optimality, with complexity $O\left(|V|^{2}\right)$, where $|V|$ is the number of nodes. The paper "Optimal proxy cache allocation for efficient streaming media distribution," by Wang, Sen, Adler, and Towsley, proposes a network bandwidth optimal solution to the streaming of a set of heterogeneous videos, from a remote server through a proxy to multiple asynchronous clients, with a low playback delay. The combination of proxy caching with different traditional server-based reactive transmission schemes such as batching, patching and stream merging, is analyzed from the transmission cost viewpoint. The authors show that even a relatively small prefix cache (10\%-20\% of the video repository) is sufficient to realize substantial savings in transmission cost, and that a carefully designed scheme can produce significant cost savings, even in predominantly unicast environments. The paper "Caching strategies in transcoding-enabled proxy systems for streaming media distribution networks," by Shen, Lee, and Basu, presents a solution to the heterogeneity of the distribution infrastructure, by enabling transcoding in network caching proxies. The authors propose to trade off computation with storage, with three different caching strategies. In each strategy, the proxy can serve low resolution stream requests by transcoding higher resolution versions stored in the cache. Performance of the different policies are analyzed under various user access patterns, and it is shown that, with a small computational load, transcoding at proxy improves the effectiveness of the cache, and decreases the user-perceived latency compared to a LRU-based caching strategy. Finally, in the paper "Smooth workload adaptive broadcast," Guo, Gao, Towsley, and Sen present a bandwidth-efficient framework for delivering popular videos to a large, asynchronous client population with time-varying demand characteristics. Two main 
components are integral parts of the framework: the innovative workload adaptive broadcast architecture and the novel flexible periodic broadcast scheme (FPB) that exhibits the smooth transition property. The architecture supports an arbitrary periodic broadcast as well as parsimonious transmission, workload adaptation, and (near-)instantaneous playback. The authors show that the bandwidth usage, proportional to the workload thanks to the adaptive broadcast, is smaller for FPB than for other schemes such as dynamic skyscraper and GDB3.

The guest editorial team of this Special Issue would like to thank all of the authors for submitting their fine work to this Special Issue. Thanks to the hard work of the reviewers who provided their expert reviews under very tight schedules, the quality of the final papers presented in this Special Issue has been greatly improved. Without their contributions, this Special Issue would not have been so timely and successful.

Finally we would like to thank Prof. Tsuhan Chen, the Editor-in-Chief, for approving the proposal of this Special Issue and for the tremendous support and guidance (including pressure) he has provided throughout the process, Prof. Ming-Ting Sun, the Past Editor-in-Chief, for his encouragement and advice in the early stage of this Special Issue. Thanks also to Deborah Tomaro from the Signal Processing Society's publication office for taking care of all details throughout the whole process of this Special Issue.

\author{
WENJUN ZENG, Lead Guest Editor \\ University of Missouri \\ Columbia, MO 65211 USA
}

KLARA NAHRSTEDT, Guest Editor
University of Illinois at Urbana-Champaign
Urbana, IL 61801 USA

PhILIP A. CHOU, Guest Editor

Microsoft Research

Redmond, WA 98052 USA

Antonio ORTEGA, Guest Editor

University of Southern California

Los Angeles, CA 90089 USA

PASCAL FrosSARD, Guest Editor

Swiss Federal Institute of Technology

Lausanne, 1015 Switzerland

HeATHer H. Yu, Guest Editor

Panasonic Information and Networking Technology Laboratory Princeton, NJ 08540 USA

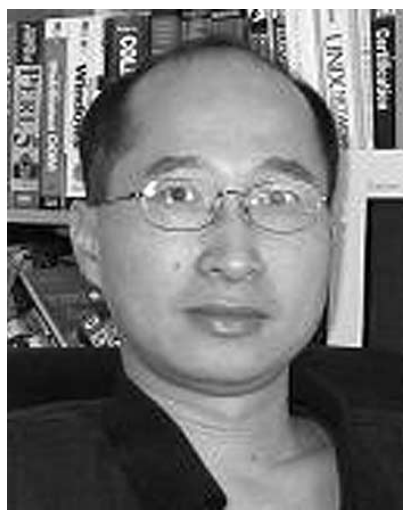

Wenjun Zeng (S'94-M'97-SM'03) received the B.E. degree from Tsinghua University, Beijing, China, in 1990, the M.S. degree from the University of Notre Dame, Notre Dame, IN, in 1993, and the Ph.D. degree from Princeton University, Princeton, NJ, in 1997, all in electrical engineering.

From 1990 to 1991, he was a Research Associate, Department of Electronic Engineering, Tsinghua University. He worked at Matsushita Information Technology Lab, Panasonic Technologies, Inc., Princeton, in the summer of 1995, and at Multimedia Communication Lab, Bell Laboratories, Murray Hill, NJ, in the summer of 1996. From 1997 to 2000, he was with Sharp Labs of America, Camas, WA. He was with PacketVideo Corporation, San Diego, CA, from December 2000 to August 2003, where he was leading R\&D projects on wireless multimedia streaming, encoder quality optimization, and digital rights management. He has been an Associate Professor with the Computer Science Department, University of Missouri-Columbia, since August 2003. His current research interests include multimedia communications and networking, content and network security, and wireless multimedia. He has been an active contributor to the JPEG 2000 image coding standard and the MPEG4 IPMP Extension standard, where four of his proposals have been adopted into the standards. He has been awarded nine patents.

Dr. Zeng has served as a special issue Guest Editor, Technical Program Committee Member, Special Session Chair, and Panel Session Organizer for several IEEE international journals and conferences. He is currently serving as the Technical Program Co-Chair of the Multimedia Communications and Home Networking Symposium, IEEE International Conference on Communications, 2005. 


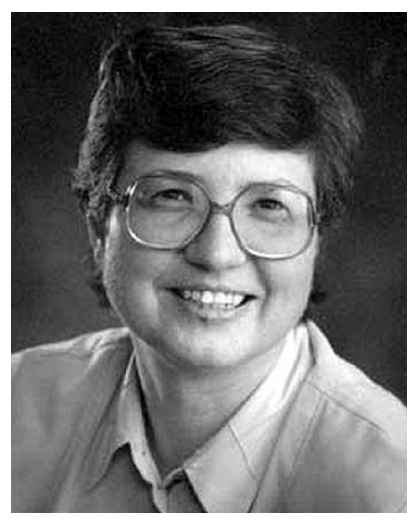

Klara Nahrstedt (S'93-M'95) received the B.A. degree in mathematics in 1984 and the M.Sc. degree in numerical analysis in 1985, both from Humboldt University, Berlin, Germany, and the $\mathrm{Ph}$.D. degree from the Department of Computer and Information Science, University of Pennsylvania, Philadelphia, in 1995.

She was a Research Scientist, Institute für Informatik, Berlin from 1985 to 1990. Currently, she is the Ralph and Catherine Fisher Associate Professor, Computer Science Department, University of Illinois at Urbana-Champaign. Her research interests are directed towards multimedia middleware systems, quality-of-service (QoS), QoS routing, QoS-aware resource management in distributed multimedia systems, and multimedia security. She is the coauthor of the widely used multimedia book Multimedia: Computing, Communications and Applications (Upper Saddle River, NJ: Prentice-Hall).

Dr. Nahrstedt was the recipient of the Early NSF Career Award, the Junior Xerox Award, and the IEEE Communication Society Leonard Abraham Award for Research Achievements. She is the editor-in-chief of ACM/Springer Multimedia Systems Journal. She is the member of the ACM.

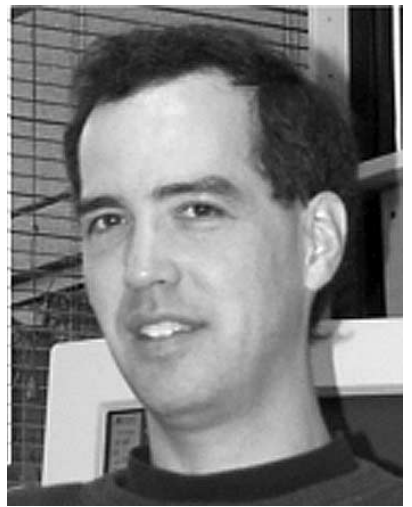

Philip A. Chou (M'88-SM'00-F'04) was born in Stamford, CT, on April 17, 1958. He received the B.S.E. degree from Princeton University, Princeton, NJ, in 1980, and the M.S. degree from the University of California, Berkeley, in 1983, both in electrical engineering and computer science, and the Ph.D. degree in electrical engineering from Stanford University, Stanford, CA, in 1988.

Since 1977, he has worked for IBM, AT\&T Bell Laboratories, Princeton Plasma Physics Lab, Telesensory Systems, Speech Plus, Hughes, Xerox, VXtreme, and Microsoft, where he was involved variously in office automation, motion estimation, character recognition, speech compression, LPC and text-to-speech synthesis, compression of digitized terrain, speech and document recognition, and multimedia network communication. His research interests are data compression, pattern recognition, and multimedia processing and communication. In 1994-1995, he was a Consulting Associate Professor at Stanford University. Since 1998, he has been an Affiliate Professor at the University of Washington, Seattle. Currently, he is with Microsoft Corporation, Redmond, WA.

Dr. Chou serves on the IEEE Technical Committee for Image and Multidimensional Signal Processing (IMDSP). From 1998 to 2001, he served on the editorial board of the IEEE TRANSACTIONS ON INFORMATION THEORY as an Associate Editor for Source Coding. He is a Fellow of the IEEE, a member of Phi Beta Kappa, Tau Beta Pi, Sigma Xi, and the IEEE Computer, Information Theory, Signal Processing, and Communications societies, and was an active member of the MPEG committee. He is the recipient, with Tom Lookabaugh, of the 1993 Signal Processing Society Paper award.

Antonio Ortega (S'91-M'95-SM'00) received the Telecommunications Engineering degree from the Universidad Politecnica de Madrid (UPM), Madrid, Spain, in 1989 and the Ph.D. degree in electrical engineering from Columbia University, New York, in 1994, where he was supported by a Fulbright scholarship.

Since 1994, he has been with the Electrical Engineering Department, University of Southern California (USC), Los Angeles, where he is currently an Associate Professor and a member of the Integrated Media Systems Center (IMSC), an NSF Engineering Research Center. He is also currently the Director of the Signal and Image Processing Institute. His research interests are in the areas of multimedia compression and communications. They include topics such as rate control and video transmission over packet wired or wireless networks, complexity optimized compression algorithms, and compression for recognition and classification applications.

Dr. Ortega received the NSF Faculty Early Career Development (CAREER) award in 1995. He is a member of SPIE and ACM. $\mathrm{He}$ is a member of the IEEE Signal Processing Society (SPS) Multimedia Signal Processing (MMSP) technical committee and Chair of the Image and Multidimensional Signal Processing (IMDSP) technical committee. He was the technical program co-chair for the 1998 Workshop on Multimedia Signal Processing and technical program co-chair of ICME 2002. He has been Associate Editor for the IEEE Transactions on IMAge Processing (1996-2000) and the IEEE Signal Processing LetTers (2000-2002), as well as member of the board of governors of the IEEE SPS (2002). He received the 1997 IEEE Communications Society Leonard G. Abraham Prize Paper Award and the IEEE Signal Processing Society 1999 Magazine Award. 


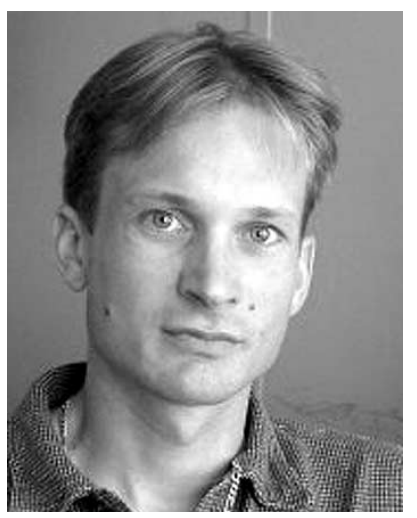

Pascal Frossard (M'01) received the M.S. and Ph.D. degrees, both in electrical engineering, from the Swiss Federal Institute of Technology (EPFL), Lausanne, Switzerland, in 1997 and 2000, respectively.

He is an Assistant Professor, supported by the Swiss National Science Foundation, at the EPFL, Lausanne. Between 2001 and 2003, he was a Member of the Research Staff, IBM T. J. Watson Research Center, Yorktown Heights, NY, where he worked on media compression, streaming technologies and delivery architectures. His main research interests currently include media information coding and delivery, joint source and channel coding, and adaptive media streaming algorithms. He is a member of the Editorial Board of the EURASIP Journal of Signal Processing.

Dr. Frossard was the general chairman of the IEEE International Conference on Multimedia and Expo in 2002 (ICME 2002). He served as Associate Program Committee Member of ACM Multimedia 2002, as Technical Program Area Chair of IEEE ICME 2004, and in the technical committee of several international conferences. He serves as vice-chair of the IEEE COMSOC Multimedia Communications Technical Committee, and is a member of the IEEE Multimedia Signal Processing technical committee.

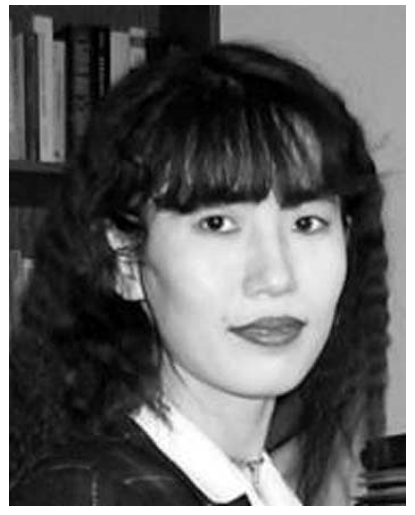

Heather H. Yu (S'97-A'98) received the B.S. degree from Peking University, Beijing, China, and the M.A. and Ph.D. degrees from Princeton University, Princeton, NJ, all in electrical engineering.

In 1998, she joined Panasonic, where she is a Senior Scientist at Panasonic Information and Networking Technologies Laboratory, Princeton. Her major focus is multimedia communication and multimedia information access R\&D. She has served as reviewer for many renowned international journals in the area of multimedia communication and processing. She has published nearly 50 technical papers, holds three U.S. patents, and has more then 20 patents pending in the multimedia communication and multimedia information access area.

Dr. Yu serves as Chair of IEEE COMSOC Multimedia Communications Technical Committee, an Associate Editor for IEEE TRANSACTIONS ON MulTIMEDIA, Editor for ACM Computers in Entertainment and IEEE MulTIMEDIA, Guest Editor of PROCEEDINGS OF THE IEEE special issue on Multimedia Security for Digital Rights Management, Conference Steering Committee Member of IEEE ICME and IEEE CCNC, Technical Program Co-Chair of IEEE ICC2004 (International Conference on Communications) Multimedia Technologies and Services Symposium, and IEEE ICC2005 Multimedia and Home Networking Symposium, Conference Technical Program Vice Co-Chair of IEEE ICME2004 (International Conference on Multimedia and Expo 2004). From 1998 to 2002, she served as conference technical program chair, associate chair, session chair, technical committee member, best paper award committee member, keynote speaker, panelist, panel chair, and steering committee member for many conferences. 